\title{
A UTILIZAÇÃO DO BALANÇO HÍDRICO COMO FERRAMENTA DE ANÁLISE AMBIENTAL DO MUNICÍPIO DE GARANHUNS/PE
}

\section{THE USE OF WATER BALANCE AS A TOOL FOR ENVIRONMENTAL ANALYSIS OF GARANHUNS/PE}

\author{
Fernando Henrique Vidal França ${ }^{1}$ \\ ${ }^{1}$ Universidade Estadual Paulista - UNESP, Faculdade de Tecnologia, Mestrado \\ Profissional de Geografia e Gestão Empresarial, Presidente Prudente, SP. \\ E-Mail: fernandoengamb@yahoo.com.br
}

RESUMO - O conjunto de dados sobre precipitação e evapotranspiração de uma determinada localidade possui importância reconhecida para a avaliação da disponibilidade de água para as mais diversas áreas e setores, tendo ainda maior relevância em regiões com histórico de episódios de escassez. No presente trabalho desenvolvemos um estudo sobre a situação hídrica do município de Garanhuns, adotando por base os dados relacionados ao balanço hídrico do período de 1998 a 2016, tratados pelo método ThornthwaiteMather. A condição de deficiência hídrica identificada referente a este intervalo temporal demonstra a relevância da utilização do balanço hídrico como ferramenta de análise ambiental para subsidiar as atividades de planejamento e gestão dos recursos hídricos.

Palavras-chave: precipitação; evapotranspiração; recursos hídricos; gerenciamento

ABSTRACT - The dataset on precipitation and evapotranspiration of a given locality has a recognized importance for the evaluation of water availability for the most diverse areas and sectors, and is even more relevant in regions with a history of shortage episodes. In the present work we developed a study on the water situation of the municipality of Garanhuns, based on data related to the water balance from 1998 to 2016, treated by the Thornthwaite-Mather method. The identified water deficiency condition related to this time interval demonstrates the relevance of using the water balance as an environmental analysis tool to subsidize the planning and management of water resources.

Keywords: precipitation; evapotranspiration; water resources; management 


\section{INTRODUÇÃO}

As condições climáticas de precipitação e evapotranspiração juntamente com as condições físicas como o relevo, a geologia, a geomorfologia e os solos, a cobertura vegetal e o uso da terra, e até mesmo as ações antrópicas sobre o sistema fluvial formam um conjunto de dados que afetam de forma dinâmica a variabilidade hidrológica e a disponibilidade de água em uma determinada localidade (TUCCI, 2000).

Diante do crescimento populacional e das imposições por um crescimento econômico contínuo, a tendência da intensificação dos múltiplos usos da água é apenas de aumento, sobre o qual pontua Galvão (2014), desacompanhado do respectivo desenvolvimento social e causador de grande pressão sobre os recursos hídricos pela crescente demanda por água aliada à sucessiva degradação das fontes de abastecimento.

O entendimento desta dinâmica e a compreensão do estreito relacionamento entre as diversas variáveis envolvidas corroboram com as atividades de análise ambiental e também contribuem para a elaboração de planos e projetos visando melhorias no aproveitamento e no controle da utilização dos recursos hídricos, justificadamente imprescindíveis em regiões com menor disponibilidade de água.

O presente trabalho caracteriza a situação hídrica do município de Garanhuns no período de 1998 e 2016, possuindo como principais objetivos: 1) a obtenção dos dados de precipitação e temperatura do ar de uma determinada região em um intervalo temporal definido; 2) a realização dos cálculos das médias anuais dos referidos dados e de outros dados decorrentes da manipulação das informações primárias; 3) a elaboração do balanço hídrico da área delimitada para o estudo e de seu respectivo gráfico; 4) a análise interpretativa dos dados levantados.

\section{METODOLOGIA}

Diante dos objetivos estabelecidos, primeiramente realizamos uma pesquisa prévia sobre as principais informações relativas à área de estudo, sendo tais dados principalmente encontrados no relatório elaborado pelo Serviço Geológico do Brasil (CPRM/PRODEEM) referente ao diagnóstico do município de Garanhuns (2005).
Já os dados da localidade sobre precipitação e temperatura do ar relativos ao intervalo temporal de 1998 a 2016 foram extraídos da base específica do NOAA (National Oceanic and Atmospheric Administration) por meio do aplicativo online Giovanni da NASA (National Aeronautics and Space Administration).

Depois de extraídos da referida plataforma os dados diários de precipitação da série TRMM e os dados de temperatura do ar da série MERRA, estes foram importados para o software Excel, onde foram calculadas as respectivas médias anuais de precipitação e também os valores de evapotranspiração potencial e real para cada ano pelo método de Thornthwaite-Mather (1955), sendo ainda realizadas a execução do balanço hídrico e sua respectiva representação gráfica.

Por fim, com a organização dos dados, realizamos as análises das tabelas e do produto gráfico confeccionado e tecemos as considerações a respeito da situação hídrica do município de Garanhuns no período determinado.

\subsection{Caracterização da área de estudo}

O município de Garanhuns está localizado na Mesorregião Agreste Meridional e na Microrregião Garanhuns do estado de Pernambuco, ocupando uma área de $465,8 \mathrm{~km}^{2}$, correspondendo a $0,47 \%$ do estado pernambucano. Sua sede, com altitude aproximada de $842 \mathrm{~m}$, possui como coordenadas geográficas 08953'25" de Latitude Sul e 36229'34" de Longitude Oeste, ficando a uma distância de 228,8 km da capital Recife.

Encontra-se ainda inserido nos domínios da Bacia Hidrográfica do Rio Mundaú, conforme apresentado na Figura 1, tendo como principais tributários os rios Mundaú, Canhoto e Inhaúma. Todos os cursos d'água no município têm regime de escoamento intermitente com padrão de drenagem dendrítico (Mascarenhas \& Outros, 2005). 
Figura 1. Mapa Hidrológico dos Rios Paraíba e Mundaú

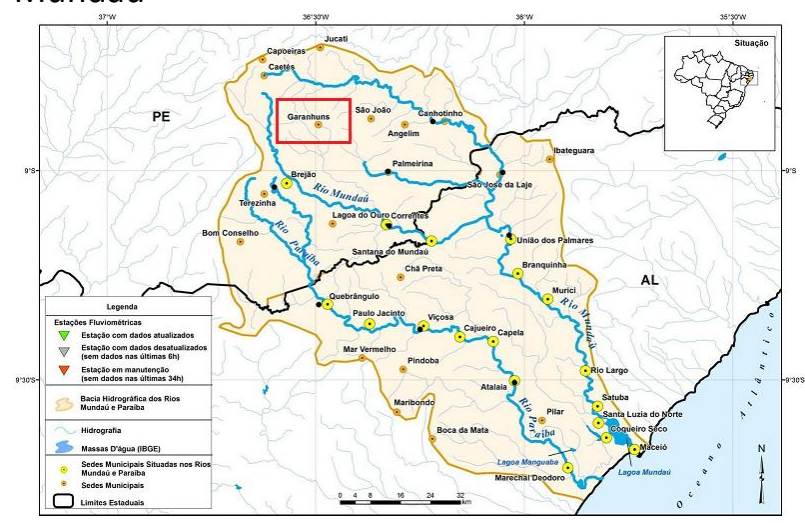

Fonte: ANA, 2017.

Seu relevo faz parte da unidade das Superfícies Retrabalhadas, formada por áreas com relevo bastante dissecado e vales profundos. O clima é do tipo Tropical Chuvoso com verão seco. A vegetação é predominantemente do tipo Floresta subperenifólia com partes de hipoxerófila.

Os solos dessa unidade geoambiental são representados pelos Latossolos nos topos planos, profundos e bem drenados, pelos Podzólicos nas vertentes íngremes, sendo pouco a medianamente profundos e drenados, e ainda, pelos Gleissolos de Várzea nos fundos de vales estreitos, com solos orgânicos e encharcados.

O município de Garanhuns está totalmente inserido no Domínio Hidrogeológico Fissural, formado de rochas do embasamento cristalino que englobam o subdomínio de rochas metamórficas constituído do Complexo Belém do São Francisco e do Complexo Cabrobó e o subdomínio de rochas ígneas dos Granitóides.

\section{RESULTADOS}

Os resultados a seguir elencados derivam dos dados obtidos da base de dados atmosféricos e oceânicos norte-americana (NOAA) pelo aplicativo Giovanni mantido pela agência espacial do mesmo país (NASA), tomando-se um quadrilátero de $0,2^{\prime}$ para as coordenadas de Garanhuns/PE (Latitude 08ㅇ 53' 25" e Longitude 36 - 29' 34") referente ao período entre os anos de 1998 e 2016.

Nesta perspectiva, por meio da análise dos dados relativos às precipitações e temperatura do ar constantes das tabelas 1 e 2 apresentadas a seguir, não foi possível identificar períodos sazonais bem definidos, tendo em vista o registro de grandes variações entre meses adjacentes (independentemente do ano) e até mesmo quando um único mês é observado isoladamente entre anos sequenciais, não sendo diferenciados períodos quentes e chuvosos de outro mais amenos e secos, dificultando a possibilidade de correlação com temperaturas na área delimitada. 
Tabela 1. Médias mensais e anuais de precipitação do município de Garanhuns (1998 a 2016).

\begin{tabular}{ccccccccccccc}
\hline ANO/MÊS & JAN & FEV & MAR & ABR & MAI & JUN & JUL & AGO & SET & OUT & NOV & DEZ \\
\hline $\mathbf{1 9 9 8}$ & 21,56 & 9,18 & 19,20 & 51,72 & 50,67 & 71,62 & 44,91 & 28,80 & 31,16 & 30,49 & 35,81 & 132,21 \\
$\mathbf{1 9 9 9}$ & 31,03 & 37,00 & 25,22 & 21,16 & 87,56 & 41,86 & 91,08 & 48,11 & 36,27 & 82,32 & 19,95 & 57,54 \\
$\mathbf{2 0 0 0}$ & 87,87 & 70,37 & 99,01 & 96,22 & 111,26 & 107,10 & 75,34 & 96,56 & 56,79 & 4,41 & 17,79 & 114,84 \\
$\mathbf{2 0 0 1}$ & 14,86 & 44,56 & 94,23 & 45,81 & 25,87 & 76,59 & 27,54 & 14,94 & 26,94 & 132,65 & 19,41 & 75,73 \\
$\mathbf{2 0 0 2}$ & 222,03 & 54,33 & 85,47 & 29,70 & 100,08 & 63,54 & 15,66 & 13,68 & 22,12 & 49,89 & 45,36 & 95,33 \\
$\mathbf{2 0 0 3}$ & 48,86 & 59,23 & 62,42 & 56,07 & 63,53 & 20,16 & 31,05 & 93,61 & 113,40 & 59,79 & 64,62 & 87,23 \\
$\mathbf{2 0 0 4}$ & 343,13 & 111,26 & 51,12 & 33,30 & 40,50 & 80,55 & 0,00 & 10,35 & 39,96 & 18,16 & 22,04 & 32,69 \\
$\mathbf{2 0 0 5}$ & 33,28 & 71,35 & 144,16 & 42,39 & 165,06 & 82,80 & 38,16 & 0,00 & 26,92 & 102,13 & 13,67 & 100,63 \\
$\mathbf{2 0 0 6}$ & 28,37 & 69,06 & 105,03 & 87,14 & 119,16 & 113,94 & 3,15 & 0,00 & 53,23 & 11,16 & 72,65 & 40,87 \\
$\mathbf{2 0 0 7}$ & 33,21 & 201,17 & 69,02 & 88,18 & 23,76 & 10,17 & 26,37 & 40,14 & 59,04 & 89,75 & 48,50 & 68,70 \\
$\mathbf{2 0 0 8}$ & 105,66 & 76,07 & 307,92 & 64,40 & 172,62 & 8,73 & 0,00 & 45,54 & 14,58 & 22,02 & 4,29 & 64,25 \\
$\mathbf{2 0 0 9}$ & 21,15 & 81,77 & 29,12 & 161,88 & 176,56 & 78,03 & 2,16 & 5,22 & 0,00 & 19,25 & 0,00 & 54,11 \\
$\mathbf{2 0 1 0}$ & 93,54 & 83,55 & 100,03 & 193,79 & 8,28 & 416,52 & 0,09 & 0,00 & 1,89 & 68,80 & 0,00 & 39,44 \\
$\mathbf{2 0 1 1}$ & 67,77 & 52,11 & 93,58 & 121,52 & 116,37 & 6,48 & 5,67 & 12,51 & 0,00 & 6,80 & 27,14 & 12,10 \\
$\mathbf{2 0 1 2}$ & 48,53 & 44,06 & 9,70 & 16,05 & 33,42 & 6,48 & 31,59 & 8,01 & 76,95 & 120,74 & 6,58 & 37,99 \\
$\mathbf{2 0 1 3}$ & 17,42 & 4,01 & 9,39 & 58,41 & 60,39 & 28,17 & 51,30 & 0,00 & 22,88 & 107,35 & 30,20 & 106,43 \\
$\mathbf{2 0 1 4}$ & 10,12 & 46,98 & 57,98 & 122,49 & 51,03 & 0,00 & 101,49 & 0,54 & 19,26 & 0,00 & 32,11 & 32,84 \\
$\mathbf{2 0 1 5}$ & 19,43 & 70,63 & 29,44 & 19,43 & 51,12 & 26,73 & 19,89 & 0,00 & $\mathbf{2 7 , 7 9}$ & 15,49 & 5,79 & $\mathbf{7 3 , 7 5}$ \\
$\mathbf{2 0 1 6}$ & 166,54 & 54,90 & 44,27 & 0,00 & 89,64 & 34,90 & 21,37 & 12,06 & 5,37 & 0,42 & 0,58 & ------ \\
\hline MÉDIA & $\mathbf{7 4 , 4 4}$ & $\mathbf{6 5 , 3 5}$ & $\mathbf{7 5 , 6 0}$ & $\mathbf{6 8 , 9 3}$ & $\mathbf{8 1 , 4 1}$ & $\mathbf{6 7 , 0 7}$ & $\mathbf{3 0 , 8 9}$ & $\mathbf{2 2 , 6 4}$ & $\mathbf{3 3 , 4 0}$ & $\mathbf{4 9 , 5 6}$ & $\mathbf{2 4 , 5 5}$ & $\mathbf{6 8 , 1 5}$ \\
\hline
\end{tabular}

Fonte: National Oceanic and Atmospheric Administration (NOAA) - Série TRMM

Tabela 2. Evapotranspiração média mensal do período 1998-2016 do município de Garanhuns.

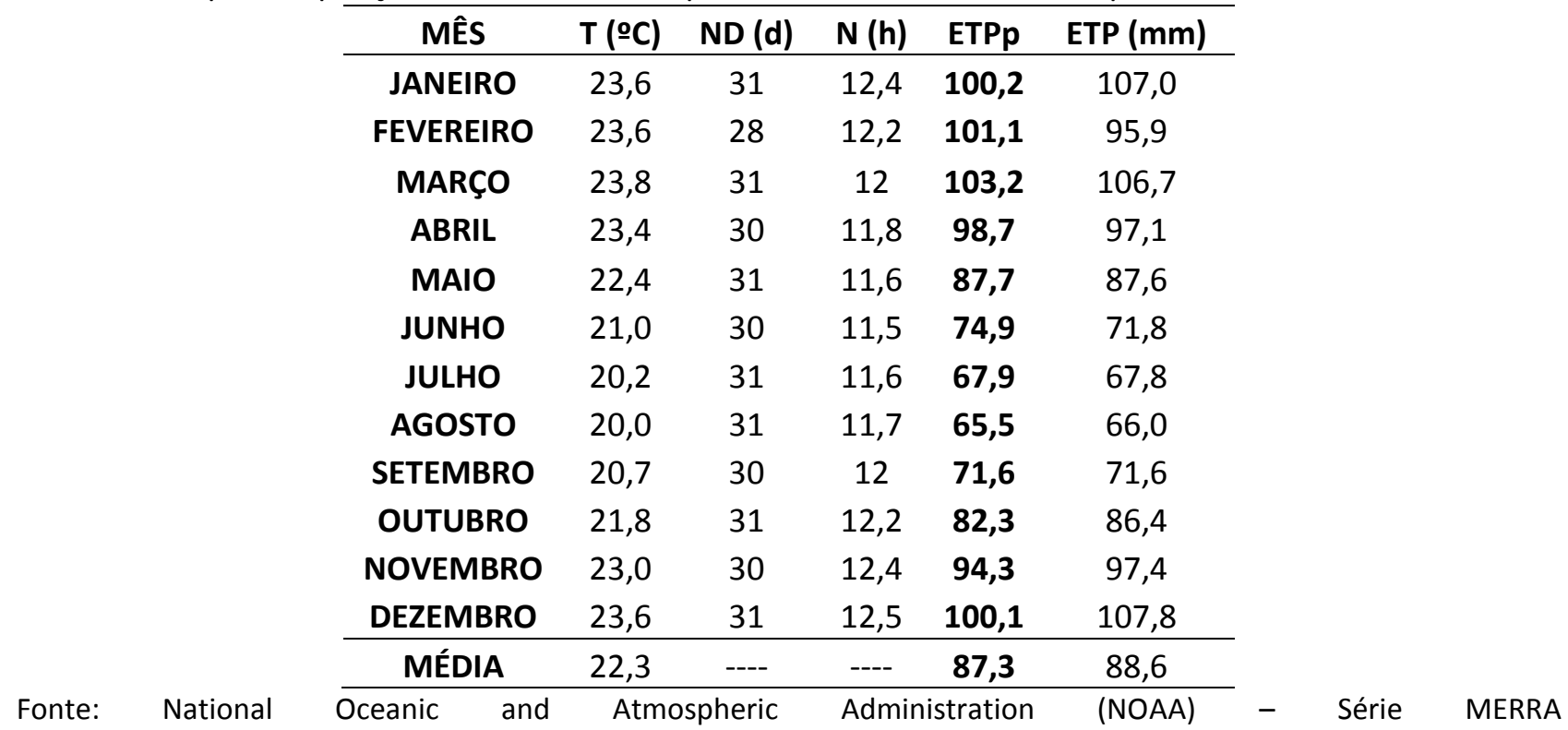


Tabela 3. Balanço Hídrico Normal de Garanhuns (Método Thornthwaite-Mather)

\begin{tabular}{ccccccccccccc}
\hline \multicolumn{4}{l}{ Local: Garanhuns/PE } & \multicolumn{4}{c}{ Latitude: 8953'S } & \multicolumn{3}{c}{ CAD = 125 } & \multicolumn{3}{c}{ Período: 1998-2016 } \\
\hline MÊS & P & ETP & P - ETP & NA & ARM & ALT & ETR & DEF & EXC & REP & RET \\
JAN & 74 & 107 & -33 & -866 & $\mathbf{0}$ & 0 & 74 & $\mathbf{3 3}$ & 0 & 0 & 0 \\
FEV & 65 & 96 & -31 & -897 & $\mathbf{0}$ & 0 & 65 & $\mathbf{3 1}$ & 0 & 0 & 0 \\
MAR & 76 & 107 & -31 & -928 & $\mathbf{0}$ & 0 & 76 & $\mathbf{3 1}$ & 0 & 0 & 0 \\
ABR & 69 & 97 & -28 & -956 & $\mathbf{0}$ & 0 & 69 & $\mathbf{2 8}$ & 0 & 0 & 0 \\
MAI & 81 & 88 & -6 & -962 & $\mathbf{0}$ & 0 & 81 & $\mathbf{6}$ & 0 & 0 & 0 \\
JUN & 67 & 72 & -5 & -967 & $\mathbf{0}$ & 0 & 67 & $\mathbf{5}$ & 0 & 0 & 0 \\
JUL & 31 & 68 & -37 & -1004 & $\mathbf{0}$ & 0 & 31 & $\mathbf{3 7}$ & 0 & 0 & 0 \\
AGO & 23 & 66 & -43 & -1047 & $\mathbf{0}$ & 0 & 23 & $\mathbf{4 3}$ & 0 & 0 & 0 \\
SET & 33 & 72 & -38 & -1085 & $\mathbf{0}$ & 0 & 33 & $\mathbf{3 8}$ & 0 & 0 & 0 \\
OUT & 50 & 86 & -37 & -1122 & $\mathbf{0}$ & 0 & 50 & $\mathbf{3 7}$ & 0 & 0 & 0 \\
NOV & 25 & 97 & -73 & -1195 & $\mathbf{0}$ & 0 & 25 & $\mathbf{7 3}$ & 0 & 0 & 0 \\
DEZ & 68 & 108 & -40 & -1235 & $\mathbf{0}$ & 0 & 68 & $\mathbf{4 0}$ & 0 & 0 & 0 \\
\hline MÉDIA ANUAL & 662 & 1063 & -401 & ------ & ------ & 0 & 662 & $\mathbf{4 0 1}$ & 0 & 0 & 0 \\
\hline
\end{tabular}

Já no balanço hídrico normal demonstrado na tabela 3 e na sua respectiva representação gráfica apresentada na Figura 2, fica evidenciada uma grave situação de deficiência hídrica do município de Garanhuns no intervalo temporal considerado.

Esta condição crítica foi influenciada consideravelmente pelos registros de precipitação com valores muito baixos e até mesmo nula em diversos meses, refletindo uma grande e constante preocupação não só deste município pertentence ao estado de Pernambuco como também trata-se de uma questão regional em relação à disponibilidade de água.

Figura 2. Gráfico do Balanço Hídrico de Garanhuns (1998 a 2016).

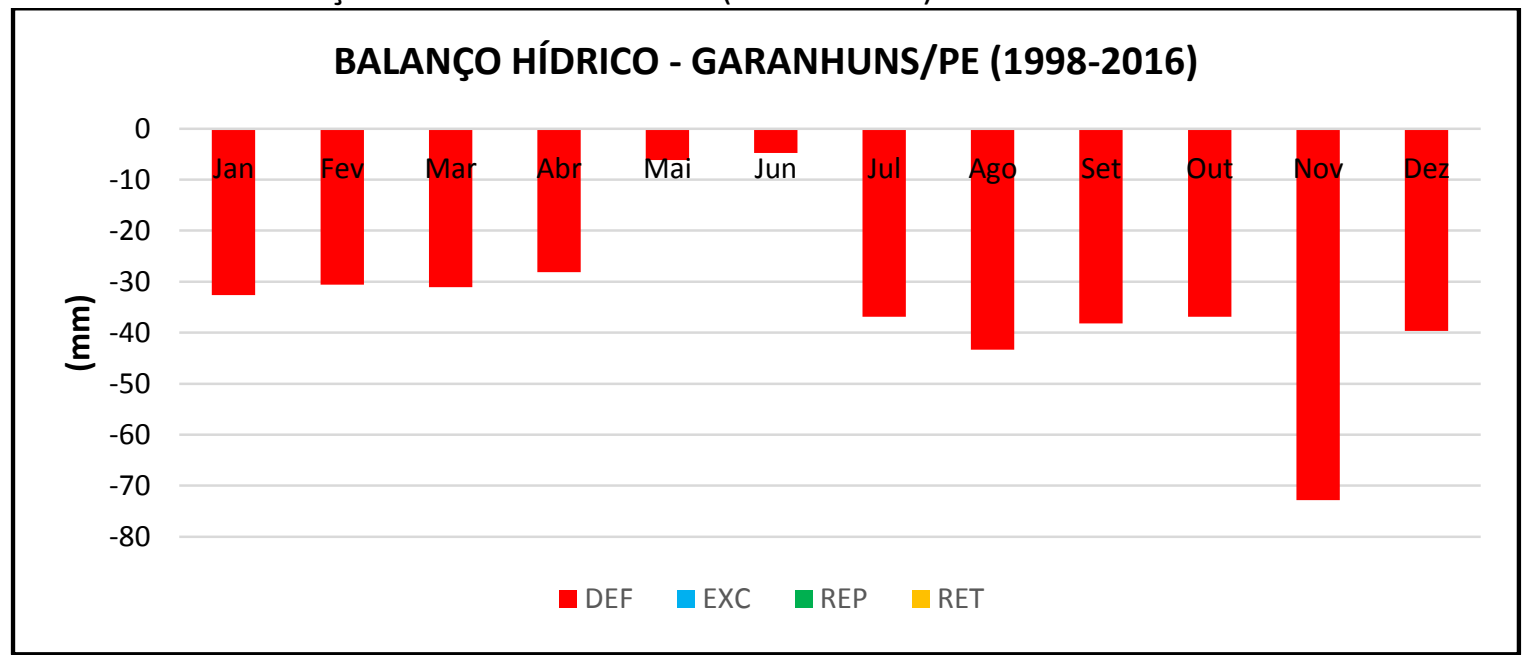

\section{DISCUSSÃO}

De acordo com Andrade \& Rocha (2011), dentre as possibilidades de influências nos climas regionais, acarretando mudanças no regime de chuvas e estiagens, e alterando, por conseguinte, o regime das vazões hidrológicas, não podem ser descartados os diferentes tipos de usos do solo, os quais também podem interferir nos regimes de chuvas de abrangência regional.

A situação identificada em relação ao município de Garanhuns no período de 1998 a 2016 reflete baixos registros de precipitação combinado com altos valores de evapotranspiração resultando num quadro de expressiva deficiência hídrica, por não ter havido 
(numericamente) armazenamento de água no solo no período compreendido entre os anos de 1998 e 2016, acarretando, por consequência, valores nulos de retirada e reposição de água do solo, posto que estes dependem diretamente das diferenças obtidas entre os valores de armazenamento.

Devido à peculiaridade da área delimitada para o presente estudo, os valores obtidos relativos à precipitação, evapotranspiração potencial e evapotranspiração real, demonstram que, caso tenha havido alguma quantidade de armazenamento de água no solo no intervalo temporal considerado, esta foi ínfima comparada ao panorama geral, sendo, por tal motivo, considerada desprezível em relação ao conjunto de dados e às especificidades e características do local em análise.

Para uma análise mais completa sobre a situação hídrica do município de Garanhuns, este trabalho propõe como desdobramento o estudo de uma série histórica mais longa, bem como a realização de análises comparativas com municípios vizinhos inseridos em sua mesma região e até mesmo pertencentes a outras regiões, mas que possuam similaridades em termos de registros de precipitação e evapotranspiração, podendo-se ainda ter seu escopo aumentado para a realização de uma investigação sobre os impactos decorrentes das atividades relacionadas ao uso do solo.

\section{CONSIDERAÇÕES FINAIS}

O balanço hídrico de Garanhuns no intervalo temporal 1998-2016 demonstrou como característica da área delimitada para a realização do presente estudo uma expressiva deficiência hídrica, em virtude de que os registros de precipitação foram, em todo o período, insuficientes para se concretizasse um mínimo possível de armazenamento de água no solo, estando tais valores significativamente abaixo da quantidade necessária para suprir a demanda hídrica local.

Nesta perspectiva, a utilização do balanço hídrico configura-se como importante como ferramenta de análise ambiental, em virtude de sua contribuição como fornecedora de subsídios para avaliação da situação hídrica de uma localidade, para o monitoramento e o planejamento relativo à disponibilidade de água e ainda contribui com as tomadas de decisões em relação à racionalização do uso da água e à sua preservação.

\section{AGRADECIMENTOS}

Agradecimentos ao professor Dr. José Tadeu Garcia Tommaselli, docente do Departamento de Geografia da Universidade Estadual Paulista, campus de Presidente Prudente pelas orientações, correções e sugestões, estas valiosas contribuições para o desenvolvimento deste trabalho.

\section{REFERÊNCIAS}

ANA - Agência Nacional de Águas. Portal Eletrônico Institucional - Sala de Situação / Sistema de Acompanhamento de Reservatórios (SAR).

<http://www2.ana.gov.br/Paginas/servicos/salad esituacao/default.aspx>. Acesso em: 02/04/2017.

ANDRADE, L. F.; ROCHA, P. C. Estudo Comparativo das Vazões dos Rios Aguapeí e Peixe. Tupã: Anais do Fórum Ambiental da Alta Paulista, 2011. v.7, n.2.

GALVÃO, A.C.F. Estudos Relativos às Mudanças Climáticas e Recursos Hídricos para Embasar o Plano Nacional de Adaptação às Mudanças Climáticas - Eixo 3: Instrumentos de Gestão de Recursos Hídricos. Brasília: CGEE - Centro de Gestão e Estudos Estratégicos, 2014.

MASCARENHAS, J. C.; BELTRÃO, B. A.; SOUZA JUNIOR, L. C.; GALVÃO, M. J. T. G.; PEREIRA, S. N.; MIRANDA, J. L. F. Diagnóstico do Município de Garanhuns/PE - Projeto Cadastro de Fontes de Abastecimento por Água Subterrânea. Recife: Serviço Geológico do Brasil - CPRM/PRODEEM, 2005.

THORNTHWAITE, C. W.; MATHER, J. R. The Water Balance. Centerton: Drexel Institute of Technology - Laboratory of Climatology, 1955.

TUCCI, C. E. M. Regionalização de Vazões. Rio Grande do Sul: Revista Universidade, 2002.

TUNDISI, J. G. Água no Século XXI: Enfrentando a Escassez. São Paulo: RiMa, 2005. 2ª ed. 